\title{
AS RELAÇÕES ENTRE CONFIANÇA, VALOR E LEALDADE NO VAREJO FARMACÊUTICO DE PARANAIIBA
}

\author{
1- Dalton de Sousa \\ Mestre em Administração pela Faculdade Cenecista de Varginha (FACECA), Brasil. \\ Professor Assistente da Universidade Federal de Mato Grosso do Sul (UFMS), Brasil. \\ dalton.sousa@gmail.com \\ http://lattes.cnpq.br/0913461440911175
}

\section{2- Leandro Canton da Silva}

Graduado em Administração pela Universidade Federal de Mato Grosso do Sul (UFMS), Brasil. canton.adm@gmail.com http://lattes.cnpq.br/7073965099090477

\section{3- Flávia Rezende Andrade Sousa}

Graduanda em Administração pela Universidade Federal de Mato Grosso do Sul (UFMS), Brasil. fla.rezende@gmail.com http://lattes.cnpq.br/4505643401456908

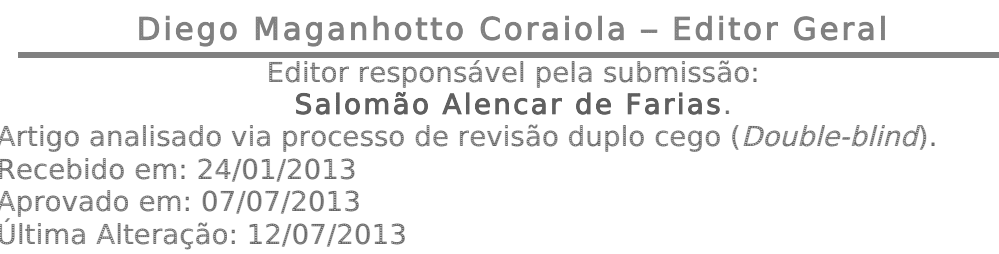

\footnotetext{
* Contato Principal: Rua Pedro Pedrossian, 725. Câmpus da UFMS. Bairro Universitário, Paranaíba - MS, Brasil, CEP: 79500000
} 


\section{AS RELAÇÕES ENTRE CONFIANÇA, VALOR E LEALDADE NO VAREJO FARMACÊUTICO DE PARANAÍBA}

\section{RESUMO}

Este trabalho analisa as relações entre confiança, valor e lealdade no varejo farmacêutico de Paranaíba (MS). Parte-se do pressuposto que o relacionamento de longo prazo com clientes certos confere diferencial estratégico para as organizações. Aplicou-se um survey a 269 consumidores de farmácias entre março e abril de 2012. Foram propostas treze hipóteses, sendo seis confirmadas e sete rejeitadas. Observou-se que a competência operacional dos funcionários de contato leva à confiança nesses mesmos funcionários, que a confiança nos funcionários de contato leva a valor e à confiança nas políticas e práticas gerenciais. Verificou-se ainda que a competência operacional das políticas e práticas gerenciais leva à confiança nas políticas e práticas gerenciais, que a confiança nas políticas e práticas gerenciais leva a valor. Por fim, o valor percebido relacionase positiva e significativamente com as intenções de lealdade do consumidor.

Palavras-chave: Confiança; Valor; Lealdade.

\section{THE RELATIONSHIPS BETWEEN TRUST, VALUE AND LOYALTY IN RETAIL PHARMACIES OF PARANAÍBA}

\section{ABSTRACT}

This paper analyzes the relationship between trust, value and loyalty in the retail pharmacies of Paranaíba (MS). It starts with the assumption that the long-term relationship with certain customers provides some strategic advantages for organizations. A survey was completed by 269 pharmacy customers between March and April 2012. Thirteen hypotheses were proposed; six hypotheses were confirmed, and seven were rejected. It was observed that the operational competence of the frontline employees leads to trust in those same employees, and that trust in the frontline employees leads to value and trust in the management policies and practices. It was also determined that the operational competence of the management policies and practices leads to trust in those management policies and practices, and that trust in the management policies and practices in turn leads to value. Finally, the perceived value relates positively and significantly with consumer loyalty.

Keywords: Trust; Value; Loyalty. 


\section{Introdução}

Diversos estudos vem sendo realizados no Brasil, em distintos contextos, para compreender as relações entre a confiança, o valor percebido e as intenções de lealdade dos consumidores (Kleinowski, 2009; Schwab, 2009; Sousa, Rezende, \& Oliveira; 2006, Porto, 2004). Tais estudos têm ressaltado que as relações de longo prazo com os consumidores (certos) podem representar um significativo diferencial competitivo para as organizações. Assim, o marketing de relacionamento surge como alternativa estratégica relevante nesse tempo de concorrência cada vez mais acirrada.

Essa perspectiva é adotada ao apresentar-se que o marketing de relacionamento surge para mudar paradigmas nas relações de mercado, propondo a transição do modelo baseado em trocas ocasionais para um modelo no qual os relacionamentos são firmados em longo prazo. Tal realidade é marcada pelo crescente interesse no estudo de relacionamentos duradouros, nas estratégias de manutenção de clientes e na conquista dos tão desejados clientes leais (Grönroos, 1994; Berry, 2002).

Todavia, de acordo com Kleinowski (2009), que realizou um levantamento dos estudos realizados no Brasil sobre confiança, valor e lealdade, o contexto farmacêutico ainda é pouco estudado pela academia brasileira.

Por sua vez, o modelo das relações entre confiança, valor e lealdade de Sirdeshmukh, Singh e Sabol (2002) vem sendo utilizado por diferentes autores no Brasil, em distintos setores e tem auxiliado na compreensão dos fatores que antecedem à formação da lealdade do consumidor (Schwab, 2009).

Dessa maneira, visando compreender as associações e as relações de interdependência entre os construtos confiança, valor e lealdade, neste trabalho propõe-se verificar empiricamente, no varejo farmacêutico da cidade de Paranaíba, Mato Grosso do Sul, as relações de interdependência entre confiança, valor e lealdade, com base no modelo teórico e empírico proposto por Sirdeshmukh et al (2002). Procura-se, ainda, atingir os seguintes objetivos específicos: verificar a influência do comportamento dos funcionários de contato e das políticas e práticas gerenciais sobre a confiança do consumidor; verificar a influência da confiança na lealdade do consumidor; identificar a relevância da confiança na percepção de valor para o consumidor, e; verificar a influência do valor percebido na lealdade do consumidor no varejo farmacêutico.

Assim, aplicou-se um survey a uma amostra de 269 clientes de drogarias e farmácias entre março e abril de 2012, para testarem-se treze hipóteses propostas neste estudo. Como resultado, obteve-se a confirmação de seis hipóteses e a rejeição de sete. O modelo ajustou-se bem à amostra obtida, mas, apresentou limitações quanto à explicação empírica das relações entre a confiança, valor e lealdade.

Em sua estruturação, o artigo apresenta inicialmente as bases teóricas para o desenvolvimento do trabalho. Em seguida, são apresentados os procedimentos metodológicos e as hipóteses da pesquisa. Na sequência são apresentados e discutidos os resultados e, por fim, são tecidas as considerações finais.

\section{Referencial Teórico}

Para diversos autores, o ambiente de serviços, no qual a percepção da incerteza e risco é acentuada, é mais propenso a investimentos no desenvolvimento da confiança e da lealdade do que no mercado de produtos (Murray, 1991; Rowley, \& Dawes, 2000; Bateson, \& Hoffman, 2001). Complementam Zeithaml, Parasuraman e Berry (1990) que os serviços diferem dos produtos em relação a como são produzidos, consumidos e avaliados.

Sirdeshmukh et al (2002), ao proporem o modelo empírico de inter-relação entre confiança, valor e lealdade, apresentam uma alternativa para a compreensão da configuração de relacionamentos duradouros entre prestadores de serviços e seus consumidores. Dessa forma, algumas definições teóricas relevantes serão apresentadas a seguir, para dar suporte às hipóteses de pesquisa.

\subsection{Confiança, Valor e Lealdade}

Em diversos trabalhos na área de marketing tem sido dado destaque ao estudo da confiança, seus antecedentes e suas consequências, notadamente os conceitos de relacionamentos duradouros e lealdade (Garbarino, \& Johnson, 1999; Gronröos, 2000; Reichheld, \& Schefter, 2000; Urban et al., 2000; Sirdeshmukh et al, 2002; Hennig-Thurau, Gwinner, \& Gremler, 2002).

De acordo com Singh e Sirdeshmukh (2000), o desenvolvimento da confiança é de grande relevância em trocas caracterizadas pelo alto nível de imprecisão no desempenho do prestador de serviço, o “alto grau de envolvimento" (Engel, Blackwell, \& Miniard, 2000, p. 106) e a grande interdependência, aqui entendida como a participação do consumidor no processo de troca (Porto, 2004).

Reichheld e Schefter (2000) corroboram esta ideia ao afirmarem que a confiança é o atributo mais relevante na opinião de consumidores não presenciais e estratégias que buscam a conquista da lealdade são fundamentais ao sucesso de negócios em ambiente virtual. Os consumidores virtuais buscam conveniência, empresas que ofereçam valor e possibilitem o desenvolvimento de ações que levem à lealdade. Para se tornarem competitivas as empresas devem ser orientadas para a agregação de valor, reconhecendo o cliente como o foco central numa troca (Churchill, \& Peter, 2000). Churchill e Peter (2000) ressaltam que a organização deve empreender esforços para fornecer valor superior para o cliente. 
Nesse sentido, Zeithaml (1988) conceitua valor como a diferença entre o que é recebido pelo consumidor em relação ao que é dado à empresa e afirma que a percepção de valor para o cliente é situacional, sendo influenciada pelo contexto em que o processo de troca está inserido (Porto, 2004). Na mesma direção, Sirdeshmukh et al (2002) definem valor como a percepção dos consumidores em relação à diferença entre os benefícios e os custos de manter um relacionamento com um provedor de serviços.

Um dos objetivos centrais das estratégias de marketing de relacionamento é a conquista da lealdade do consumidor, por isso conquistar sua confiança e oferecer-lhe alto valor nas trocas relacionais são condições fundamentais para essa conquista (Sirdeshmukh et al, 2002). Diferentemente, no passado, fatores como adequada localização de lojas, agressividade da força de vendas e falta de informações serviam de proteções para as empresas, possibilitando-lhes oferecer menos que o produto ou serviço de melhor qualidade, o que provocava a inércia de clientes, muitas vezes confundida com a lealdade (Reichheld, 2002).

A lealdade do consumidor tem sido apresentada, por estudos atuais de marketing, como um fator central no desenvolvimento de relações e sua conquista pode significar uma vantagem competitiva para as organizações (Oliver, 1999). Todavia, o atual contexto competitivo dificulta a conquista da lealdade pelas empresas, uma vez que os consumidores são mais sujeitos às promoções e novidades disponíveis no mercado. Ou seja, desenvolver uma estratégia para conquistar a lealdade é cada vez mais complicado num mercado com tendência ao declínio constante de consumidores leais (Oliver, 1999; Rowley, \& Dawes, 2000). Para Oliver (1999), os atuais consumidores só estão dispostos a serem leais se forem capazes de perceber que a empresa continua sendo a melhor alternativa de mercado.

Nesse sentido, faz-se importante conceituar a lealdade do consumidor e ela é definida por Oliver (1997, p. 34) como: "um profundo compromisso mantido de recomprar [...] um produto/serviço preferido consistentemente no futuro, [ ...] sem a preocupação com influências situacionais e esforços de marketing com potencial para causar mudanças de comportamento". Corroboram Bei e Chiao (2001), que a conceituam como o comprometimento em compras repetitivas de um mesmo produto ou serviço, sendo esse um comportamento não influenciável por apelos externos da concorrência.

Para Henning-Thurau et al (2002), a lealdade é mais que um processo de compra repetitivo e está relacionada à apreciação e ao comprometimento. Nesse sentido, Singh e Sirdeshmukh $(2000$, p. 161) definem a lealdade como um construto relacional, sendo "um comportamento que demonstra a intenção de manter e ampliar um relacionamento com um provedor de serviço".

Henning-Thurau et al (2002) enfatizam que o cliente leal compromete-se com a divulgação da empresa e seus produtos por meio do boca-a-boca positivo e demonstra um desejo de manter o relacionamento. Relevante contribuição, relacionada ao boca-a-boca positivo, é apresenta por Reichheld (2002, p. 32) ao afirmar que, as indicações são rentáveis no comércio tradicional, mas a internet amplifica seu efeito e afirma que o "mouse a mouse difunde-se com rapidez ainda maior que o boca a boca". Almeida e Ramos (2012) concordam e afirmam que a comunicação boca a boca on line alcança rapidamente muitos consumidores reais ou potenciais, potencializando ameaças ou oportunidades aos negócios.

\subsection{O Modelo de Inter-relação entre Confiança, Valor e Lealdade}

No modelo de inter-relação entre confiança, valor e lealdade proposto por Sirdeshmukh et al (2002), a confiança é uma variável que precede a lealdade, exercendo sobre ela influência positiva. O construto valor emerge como mediador entre a confiança e a lealdade.

Mais que verificar as consequências da confiança do consumidor, nesse modelo representadas pelo valor e lealdade, Sirdeshmukh et al (2002) propuseram verificar seus antecedentes. Porto (2004, p.49) afirma que "os autores [...] propõem que a confiança é um construto multifacetado decorrente de duas dimensões distintas que a antecedem: a confiabilidade nos funcionários de contato e a confiabilidade nas políticas e práticas gerenciais da empresa". Verifica-se no modelo que a confiança possui, em consonância com seus antecedentes, duas facetas: funcionários de contato e políticas e práticas gerenciais. No modelo, estas duas dimensões da confiabilidade são diretamente influenciadas por três diferentes construtos, que são, competência operacional, benevolência operacional e orientação para a solução de problemas. Na sequência, essas dimensões são mais bem explicadas.

\subsubsection{As dimensões precedentes à confiança}

Para Sidershmukh et al (2002), os consumidores avaliam diferentemente o comportamento dos funcionários de contato, além de serem capazes de identificar aquelas que são as políticas e as práticas gerenciais de uma organização. Para esses autores, a confiabilidade nos funcionários de contato é avaliada pelos consumidores pela observação do comportamento dos funcionários durante o encontro de serviços; já a confiabilidade nas políticas e práticas gerenciais é avaliada pelo julgamento dos consumidores em relação às políticas e práticas que direcionam o posicionamento da empresa. Para Sidershmukh et al (2002), a empresa demonstra confiabilidade quando o comportamento dos funcionários e as políticas e práticas estabelecidas indicam motivação e direcionamento a favor dos interesses do consumidor.

Almeida e Ramos (2012) afirmam que a confiança é a variável mais aceita como base para as relações de troca. Estes autores concluíram, após um estudo experimental em que consumidores foram expostos a 
situações de reclamações on line sobre produtos que os mesmos possuíam, que estas reclamações afetaram a lealdade.

\subsubsection{Os funcionários de contato e as políticas e práticas gerenciais}

A lealdade a ser conquistada, na visão de Reichheld (2002), deve ser não somente a dos clientes, mas também a dos funcionários. Para esse autor, a verdadeira lealdade dos funcionários é constituída pela responsabilidade pessoal e pelo seu papel no desenvolvimento de relacionamentos bem sucedidos, de elevado valor para todas as partes.

Mattila (2001) sugere que a chave do desenvolvimento de adequada estratégia de marketing de relacionamento é o gerenciamento das interações entre consumidores e funcionários. Acrescentam Doney e Cannon (1997) que os vendedores desenvolvem papel-chave, ao considerar-se a questão da persuasão dos consumidores na hora da compra. Para Bitner, Booms e Mohr (2001), os funcionários de contato são um diferencial estratégico para a empresa por serem uma importante fonte de informações sobre os clientes e propõem que um dos momentos mais importantes para a avaliação do serviço é o chamado "momento da verdade", em que se estabelece o contato entre o consumidor e o funcionário.

Por sua vez, as políticas e práticas gerenciais estabelecidas por uma organização constituem a outra faceta da confiança, de acordo com o modelo proposto de Sirdeshmukh et al (2002). Para os autores, sob esse aspecto, a confiabilidade na organização relaciona-se às suas políticas estabelecidas, aos contratos, termos e documentos que expressem como serão suas ações frente às diversas situações de troca. Segundo os mesmos autores, o consumidor também avalia as práticas gerenciais, expressas no cumprimento das políticas que, uma vez estabelecidas, devem ser praticadas, demonstrando ao consumidor credibilidade e confiabilidade. Lovelock e Wright (2002) concordam com a ideia de que a empresa precisa demonstrar suas políticas na prática, tornando-as operacionais.

\subsubsection{Competência operacional, benevolência operacional e orientação para solução de problemas}

A competência operacional, apresentada no modelo de Sirdeshmukh et al (2002) como influenciadora da confiabilidade dos consumidores, relaciona-se à expectativa do consumidor em relação a um desempenho competente da empresa. A percepção em relação à competência de uma organização pode ser determinante para a manutenção ou o rompimento de um relacionamento (Sirdeshmukh et al, 2002; Porto, 2004).

Nesse sentido, outros estudiosos do assunto afirmam que a perícia, conhecimento e competência técnica dos funcionários influenciam positivamente a confiança do consumidor (Doney \& Cannon, 1997). Sirdeshmukh et al (2002) enfatizam que a competência deve ser operacional.

Para Porto (2004) e Sirdeshmukh et al (2002), a benevolência é uma dimensão importante na explicação da confiança, pois é caracterizada por indicar ações favoráveis ao consumidor e que vão além do que está delineado em contratos ou padrões pré-estabelecidos. Corroborando, Jap (2001) conceitua que a benevolência é caracterizada pelos comportamentos percebidos pelo consumidor como além do previsto para determinada função, que revelam comprometimento, credibilidade e fortalecem as relações entre empresa e consumidor.

Sirdeshmukh et al (2002) propõem a "orientação para solução de problemas" como a última das dimensões que antecedem a confiança e a definem essa dimensão como "motivações para antecipar e resolver satisfatoriamente os problemas que possam surgir durante e depois de uma troca de serviços" (Sirdeshmukh et al, 2002, p. 18). Tais autores afirmam que os problemas devem ser vistos pela empresa como uma oportunidade para mostrar seu comprometimento com o serviço oferecido. Nessa direção, Maxham III e Netemeyer (2002) sustentam que os esforços de uma empresa para solucionar os problemas existentes desenvolvem a satisfação secundária, ou seja, a satisfação com a solução encontrada, fazendo com que esta se sobreponha à percepção negativa do problema inicial.

\section{Procedimentos Metodológicos}

Neste trabalho replicou-se o método utilizado por Porto (2004) e Sousa et al (2006), aplicando-se o modelo proposto por Sirdeshmukh et al (2002). Essa opção ganhou força na afirmação de Berndt e Oliveira (2005, p. 5) de que "a simples idéia de que a ciência evolui por meio de contínuas comparações é uma constante na realização de pesquisas de qualquer natureza". Assim, acredita-se que a aplicação do modelo em um contexto distinto, em outro mercado, de outra região do país, com diferente tamanho de amostra, em distinto espaço de tempo e sob a visão de outros pesquisadores poderá contribuir para a verificação do modelo adotado.

Foi realizada uma pesquisa do tipo descritiva. Para Malhotra (2001), o principal objetivo da pesquisa descritiva é descrever alguma coisa, normalmente características ou funções de mercado, sendo que tais estudos são aplicados para examinar relações entre variáveis e para fazer previsões específicas, com base em hipóteses apresentadas num modelo teórico. Para o autor, a pesquisa descritiva é formal e estruturada e a análise dos dados resultantes é feita quantitativamente.

Realizou-se um estudo transversal que promove a coleta de informações de qualquer amostra de uma população de uma única vez (Malhotra, 2001). Realizou-se estudo transversal único, no qual é "extraída somente 
uma amostra de entrevistados da população-alvo e as informações são obtidas desta amostra somente uma vez" (Malhotra, 2001, p. 109).

O método de survey foi adotado na coleta de dados, no qual "um questionário estruturado dado a uma amostra de uma população é destinado a provocar informações específicas dos entrevistados" (Malhotra, 2001, p. 179). Dessa forma, obtêm-se uma padronização no processo de coleta dos dados.

Com base em Malhotra (2001, p. 301) que propõe que “a população é a soma de todos os elementos que compartilham algum conjunto comum de características, conformando o universo para o propósito do problema de pesquisa de marketing", a população desta pesquisa foi definida como sendo consumidores de produtos farmacêuticos da cidade de Paranaíba, Mato Grosso do Sul.

\subsection{A Proposição das Hipóteses}

A seguir, são apresentados os fundamentos que, adicionalmente ao referencial teórico apresentado, nortearam a proposição das treze hipóteses de pesquisa apresentadas na Figura 1. Apresenta-se também, por meio da Figura 2, o modelo de relações entre confiança, valor e lealdade, proposto por Sirdeshmukh et al (2002), com o respectivo posicionamento das hipóteses.

Sirdeshmukh et al (2002) afirmam que a competência deve ser operacional, por meio de uma execução competente e comportamentos visíveis nos serviços em ação (resposta rápida), sendo distinta da competência inerente (conhecimento). Os autores ressaltam, ainda, que, a competência operacional é avaliada pelos consumidores por meio da observação do comportamento dos funcionários de contato e nas políticas e práticas gerenciais. Assim, Sirdeshmukh et al (2002) propõem que a avaliação da competência operacional pelo consumidor é um determinante crítico da confiança, tanto em relação aos funcionários de contato quanto às políticas e práticas gerenciais. Assim, foram propostas as Hipóteses 1 e 2, conforme a Figura 1.

\begin{tabular}{|c|l|}
\hline H1 & $\begin{array}{l}\text { A percepção do consumidor em relação à competência operacional, evidente no comportamento dos } \\
\text { funcionários de contato, influencia positivamente a confiança nos funcionários de contato. }\end{array}$ \\
\hline H2 & $\begin{array}{l}\text { A percepção do consumidor em relação à competência operacional, evidente nas políticas e práticas } \\
\text { gerenciais, influencia positivamente a confiança nas políticas e práticas gerenciais. }\end{array}$ \\
\hline H3 & $\begin{array}{l}\text { A percepção do consumidor em relação à benevolência operacional, evidente no comportamento dos } \\
\text { funcionários de contato, influencia positivamente a confiança nos funcionários de contato. }\end{array}$ \\
\hline H4 & $\begin{array}{l}\text { A percepção do consumidor em relação à benevolência operacional, evidente nas políticas e práticas } \\
\text { gerenciais, influencia positivamente a confiança nas políticas e práticas gerenciais. }\end{array}$ \\
\hline H5 & $\begin{array}{l}\text { A percepção do consumidor em relação à orientação para a solução de problemas, evidente no } \\
\text { comportamento dos funcionários de contato, influencia positivamente a confiança nos funcionários de } \\
\text { contato. }\end{array}$ \\
\hline H6 & $\begin{array}{l}\text { A percepção do consumidor em relação à orientação para a solução de problemas, evidente nas } \\
\text { políticas e práticas gerenciais, influencia positivamente a confiança nas políticas e práticas } \\
\text { gerenciais. }\end{array}$ \\
\hline H7 & $\begin{array}{l}\text { A confiança do consumidor nos funcionários de contato é positivamente influenciada pela confiança } \\
\text { nas políticas e práticas estabelecidas pela organização. }\end{array}$ \\
\hline H8 & $\begin{array}{l}\text { A confiança do consumidor nas políticas e práticas da organização é positivamente influenciada pela } \\
\text { confiança nos funcionários de contato. }\end{array}$ \\
\hline H9 & $\begin{array}{l}\text { A lealdade do consumidor em relação à empresa é positivamente influenciada pela confiança nos } \\
\text { funcionários de contato. }\end{array}$ \\
\hline H10 & $\begin{array}{l}\text { A lealdade do consumidor em relação à empresa é positivamente influenciada pela confiança nas } \\
\text { políticas e práticas gerenciais da empresa. }\end{array}$ \\
\hline H11 & \begin{tabular}{l} 
A lealdade do consumidor em relação ao provedor de serviço é positivamente influenciada pelo valor. \\
\hline H12
\end{tabular} O valor é positivamente influenciado pela confiança nos funcionários de contato. \\
\hline H13 & O valor é positivamente influenciado pela confiança nas políticas e práticas gerenciais da empresa. \\
\hline
\end{tabular}

Figura 1 - Hipóteses da pesquisa.

Fonte: Elaborada pelos autores.

A benevolência é conceituada como “os comportamentos que refletem uma motivação subjacente para colocar o interesse do consumidor acima do próprio interesse" (Sirdeshmukh et al, 2002, p. 18). Assim como na dimensão da competência, tais autores são contundentes ao dizer que a benevolência deve ser operacionalmente visível (e não somente uma motivação para a benevolência), podendo ser observada e avaliada pelos clientes. Para Sirdeshmukh et al (2002), a benevolência é uma dimensão importante e antecedente da confiança, pois é caracterizada por indicar ações favoráveis ao consumidor e que vão além do que está delineado em contratos ou padrões pré-estabelecidos. Dessa forma, a benevolência operacional, proposta por Sirdeshmukh et al (2002), influenciará positivamente a confiança do consumidor, dando origem, então, às Hipóteses 3 e 4.

Sirdeshmukh et al (2002) apresentam a orientação para a solução de problemas como a terceira dimensão a exercer influência nas políticas e práticas gerenciais e nos funcionários de contato. Dessa forma, são propostas as Hipóteses 5 e 6.

Para Sirdeshmukh el al (2002), a confiança do consumidor nos funcionários de contato e a confiança nas políticas e práticas gerenciais estabelecidas pela organização influenciam-se mutuamente. Com base em tal proposição, são apresentadas as Hipóteses 7 e 8 deste trabalho por meio da Figura 1. 
Na visão de Reichheld e Schefter (2000, p. 107) “para ganhar a lealdade de clientes, você deve primeiro ganhar sua confiança". Tal afirmativa é corroborada por Sirdeshmukh et al (2002), que propõem que a lealdade do consumidor é construto antecedido e influenciado positivamente pela confiança do consumidor. Assim, temse que a lealdade do consumidor é positivamente afetada pela confiança do consumidor, como descrito nas Hipóteses 9 e 10.

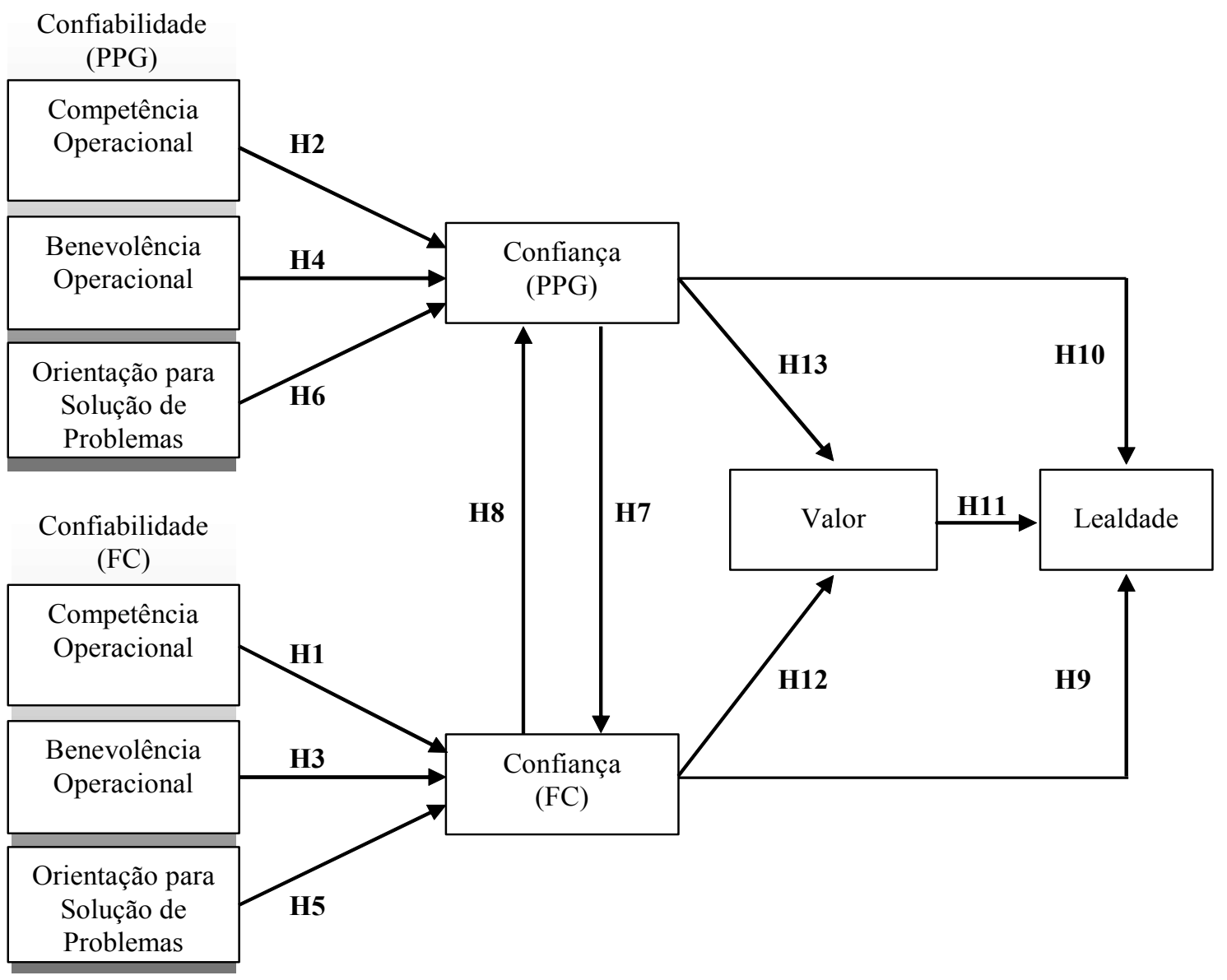

Figura 2: O modeló daś relaçõé éntre Confiança, Valor e Lealdade é as Hipótésés da Pesquiśa

Fonte: Sirdeshmukh, D. Singh, J., \& Sabol, B. (2002). Consumer Trust, Value, and Loyalty in Relational Exchanges. Journal of Marketing. 66 (1), 15-37.

PPG - Políticas e Práticas Gerenciais; FC - Funcionários de Contato.

Para Reichheld (1996, p. 3), “a criação de valor para o cliente gera lealdade e esta, por sua vez, gera crescimento, lucros e mais valor". Assim, o lucro é uma consequência da criação de valor para o consumidor e da sua lealdade. Nesse sentido, Sirdeshmukh et al, (2002) propõem que o comportamento de lealdade de um consumidor em relação à empresa cresce de acordo com o valor que a mesma pode lhe oferecer durante as trocas relacionais, sendo a confiança considerada precedente ao valor, quando há uma interação positiva com o prestador de serviço. Com base em tais pressupostos, são propostas as Hipóteses 11, 12 e 13, descritas na Figura 1 e devidamente posicionadas na Figura 2.

\subsection{Elaboração do Questionário, Obtenção da Amostra e Técnica de Análise dos Dados}

O questionário foi adaptado ao contexto deste trabalho com base na tradução para a língua portuguesa realizada por Porto (2004), do trabalho de Sirdeshmukh et al (2002). O trabalho de Sousa et al (2006) também foi consultado. Além disso, recorreu-se à versão original do instrumento, em língua inglesa.

Os construtos de confiabilidade nos funcionários de contato e confiabilidade nas políticas e práticas gerenciais foram operacionalizados por meio de nove indicadores para cada dimensão (Figura 3), que estão subdivididos em três grupos de acordo com as variáveis propostas por Sirdeshmukh et al (2002), como componentes da confiabilidade: a competência operacional, a benevolência operacional e a orientação para a solução de problemas. Tais indicadores foram avaliados pelos respondentes numa escala de cinco pontos, que variou de (1) "discordo totalmente" a (5) "concordo totalmente".

A confiança nos funcionários de contato, a confiança nas políticas e práticas gerenciais, o valor e a lealdade foram operacionalizadas por meio da utilização de quatro indicadores cada (Figura 3), cuja avaliação foi realizada utilizando-se uma escala de dez pontos. 


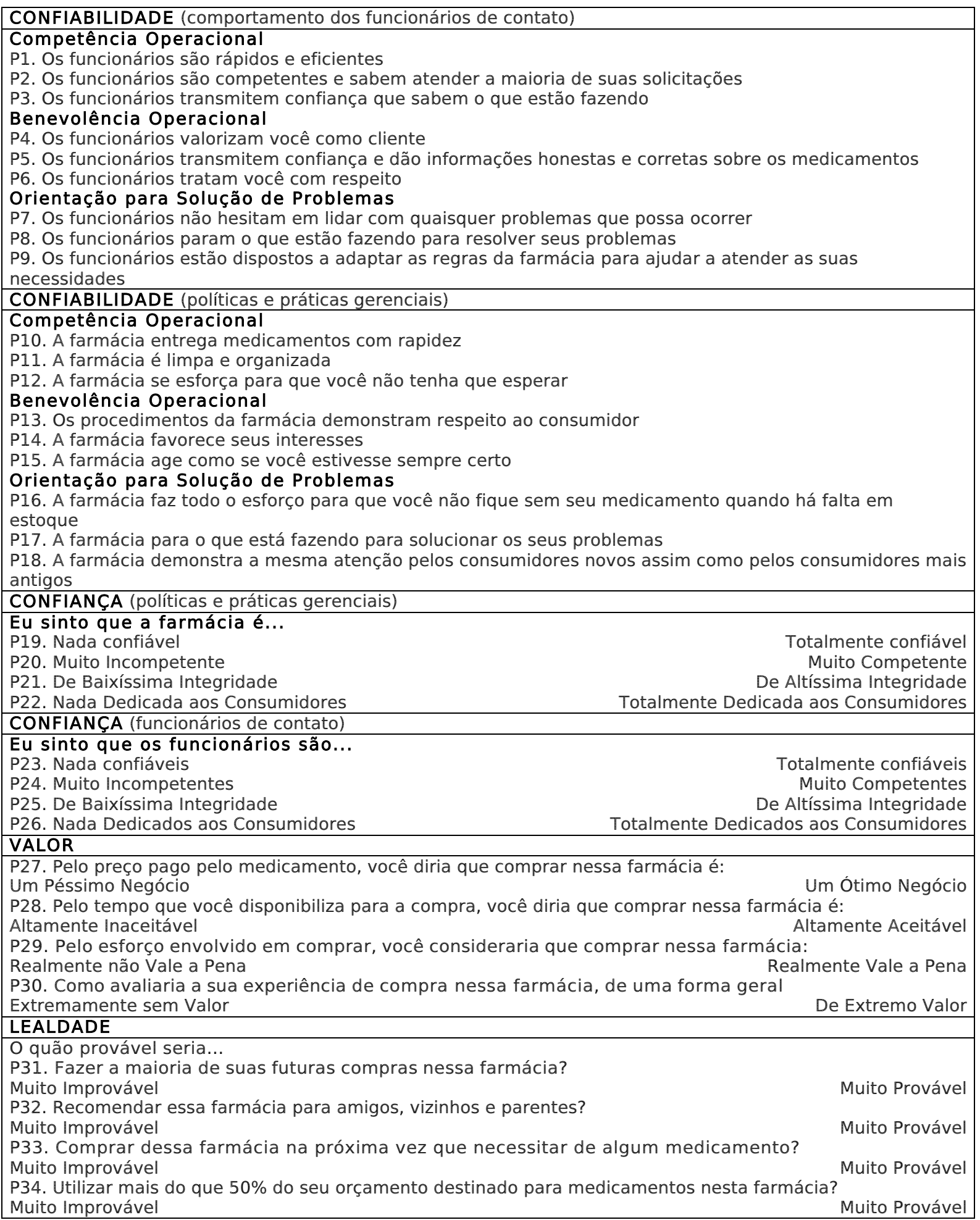
Figura 3 - Operacionalização das variáveis.

Fonte: Elaborada pelos autores.

O survey foi aplicado a uma amostra de 269 consumidores de farmácias da cidade de Paranaíba entre os meses de março e abril de 2012. Comparativamente, as amostras de Sirdeshmukh et al (2002) foram de 264 respondentes no varejo de vestuário e 113 respondentes para viagens aéreas; a amostra obtida por Porto (2004) foi de 427 respondentes no contexto da educação a distância e a de Sousa et al (2006) foi de 937 respondentes, também na educação à distância.

A amostra foi obtida por meio de entrevistas aplicadas a consumidores nas áreas de comércio varejista de maior movimentação de pessoas na cidade de Paranaíba. Caracterizando-se assim como uma amostragem não probabilística e por conveniência.

Conforme Hair, Wolfinbarger, Ortinau e Busch (2010, p. 159), “na amostragem não probabilística, a probabilidade de seleção de cada unidade amostral é desconhecida [...], o erro de amostragem é 
desconhecido". Dessa forma, o pesquisador pode, de forma arbitrária ou consciente, selecionar os elementos a serem incluídos na amostra (Hair et al, 2010; Malhotra, 2001). De acordo com Hair et al (2010), um método usual de obtenção de amostra por conveniência é realizar entrevistas em áreas com bastante tráfego de pessoas.

A técnica utilizada na avaliação do modelo proposto foi a análise fatorial confirmatória, por meio da aplicação da Modelagem de Equações Estruturais (Hair, Anderson, Tatham, \& Black, 1995). Segundo os autores, a técnica de análise fatorial confirmatória permite ao pesquisador avaliar simultaneamente um conjunto de relações múltiplas e interdependentes entre as variáveis, através de uma série de equações e regressões múltiplas, sendo particularmente adequada quando uma variável dependente torna-se independente num outro relacionamento. Conforme Marôco (2010), a modelagem de equações estruturais tem por base um quadro teórico estabelecido previamente e os dados irão confirmar ou não tal quadro. Para Marôco (2010, p. 4) “a teoria é assim o motor da análise, contrariamente ao paradigma da estatística clássica, em que os dados, e não a teoria, estão no centro do processo de análise".

Com base nas afirmações de Bagozzi e Edwards (1998) consideraram-se neste trabalho todos os construtos como sendo de primeira ordem, uma simplificação do modelo que pode ser realizada para obtenção de parcimônia na estimação dos parâmetros. Assim, de acordo com as recomendações de Bagozzi e Edwards (1998), adotou-se o processo de agregação total, em que cada construto pode ser representado pela média dos valores medidos através de seus indicadores, o que converte uma variável latente em uma variável observada.

A verificação do ajustamento do modelo foi realizada com a aplicação de algumas medidas de ajustamento propostas por Hair et al (1995). Os indicadores considerados foram empregados na pesquisa de Sirdeshmukh et al (2002), Porto (2004) e Sousa et al (2006). Porto (2004) afirma que "existem várias medidas de ajustamento, não se podendo atribuir a um único coeficiente o ajustamento de um modelo". Neste estudo, foram utilizados o Qui-quadrado sobre graus de liberdade (x2/GL) e o Root Mean Square Error of Approximation (Rmsea) como medidas absolutas de ajustamento (Hair et al, 1995). O rmsea é uma medida para corrigir a tendência que o teste de qui-quadrado apresenta em rejeitar modelos especificados com uso de grandes amostras e representa a discrepância por grau de liberdade da raiz quadrada da média dos resíduos dos modelos observados e esperados ao quadrado. Consideram-se valores entre 0,05 e 0,08 aceitáveis (Hair et al, 1995).

Além das medidas absolutas de ajustamento, foram utilizadas duas medidas comparativas de ajustamento, que comparam o modelo proposto ao modelo nulo, que possui todos os indicadores medindo perfeitamente os construtos, ou seja, apresenta valores de Qui-quadrado associados com a variância total na série de correlações ou de covariâncias (Hair et al, 1995). Foram aplicadas as seguintes medidas comparativas de ajustamento, de acordo com Hair et al (1995): Tucker-Lewis Index (TLI); Comparative Fit Index (CFI).

O software estatístico utilizado foi o Amos ${ }^{\circledR}$ 5.0.1 (Arbuckle, 1999). Com o Amos Graphics, podem-se realizar as análises a partir do diagrama, sem a necessidade de indicar as equações.

Para verificação das hipóteses foram analisadas as significâncias estatísticas e a magnitude dos parâmetros estimados, com base na análise dos t-values e das cargas fatoriais ou coeficientes de regressão padronizados $(\beta)$. Para Hair et al (1995), os t-values possibilitam identificar a significância de um parâmetro estimado e as cargas fatoriais indicam a força das relações estabelecidas. A verificação de uma carga fatorial significativa implica a consideração de que a relação entre as duas variáveis é empiricamente comprovada.

\section{Resultados e Discussão}

Primeiramente foram analisadas as medidas de ajustamento geral do modelo. Os dados da Tabela 1 demonstram bom ajustamento do modelo em estudo na amostra em questão, levando-nos a afirmar que, de forma geral, as relações propostas pelo modelo teórico estão adequadas ao contexto em que a pesquisa empírica foi desenvolvida.

Tabela 1

Ajustamento Geral do Modelo

\begin{tabular}{l|c|c}
\hline Medida & Ajustamento & Limites \\
& & Aceitação \\
\hline Qui-Quadrado (QQ) & 47,31 & - \\
Graus de Liberdade & 17,00 & - \\
QQ/GL & 2,78 & $\leq 5,00$ \\
TLI & 0,95 & $\geq 0,90$ \\
CFI & 0,98 & $\geq 0,90$ \\
Rmsea & 0,08 & $0,05 \geq$ rmsea $\leq 0,08$ \\
\hline
\end{tabular}

Nota. Fonte: Resultados da pesquisa. Elaborado pelos autores.

O qui-quadrado sobre graus de liberdade (QQ/GL) apresentou valor aceitável, sendo inferior a 5: $(47,31 / 17=2,78)$. O rmsea apresentou valor dentro do limite do intervalo de aceitação, sendo igual a 0,08 . As outras medidas apresentaram bons índices de ajustamento, sendo todas superiores a $0,90, \mathrm{CFI}=0,98, \mathrm{TLI}=$ 0,95 . 
Na Tabela 2 observam-se os dados de categorização da amostra. Verifica-se que a maioria dos entrevistados tem entre 16 e 25 anos de idade (39,03\%), são do sexo feminino (52,04\%) e tem renda de até R\$ $1.000,00(53,90 \%)$.

Os resultados para as hipóteses da pesquisa são apresentados por meio da Tabela 3, onde são encontrados os valores dos t-values, os coeficientes de regressão e as Cargas Fatoriais ou Coeficientes de Regressão Padronizados $(\beta)$, bem como as conclusões sobre a confirmação ou a rejeição das hipóteses da pesquisa.

Para a amostra deste estudo, a competência operacional mostrou-se importante para o estabelecimento da confiança nos funcionários de contato, confirmando-se assim a hipótese 1 (H1). Assim, corrobora-se o que afirmaram Sousa et al (2006), de que a competência operacional é o principal fator para o estabelecimento da confiança do consumidor em relação aos funcionários de contato.

Assim como os resultados desta pesquisa, os resultados obtidos por Porto (2004) também confirmaram apenas a Competência Operacional como antecedente positivo e significativo sobre a Confiança nos Funcionários de Contato. Dessa forma, ratifica-se a relevância da competência operacional para a manutenção ou rompimento de um relacionamento (Sirdeshmukh et al, 2002; Porto, 2004).

Todavia, os demais antecedentes dessa confiança não foram confirmados como significativos no teste das hipóteses 3 e 5 desta pesquisa. Ou seja, a benevolência operacional e a orientação para solução de problemas, expressas no comportamento dos funcionários de contato como importantes para a formação da confiança em tais funcionários, não se mostraram significativas para a amostra em questão.

Tal como no trabalho de Sousa et al (2006), confirmou-se também a hipótese 2 (H2), assim, a competência operacional, expressa nas políticas e práticas gerenciais, exerce positiva e significativa influência sobre a confiança nas políticas e práticas gerenciais.

Por outro lado, as hipóteses 4 e 6 foram rejeitadas, demonstrando que a benevolência operacional e a orientação para solução de problemas, expressas nas políticas e práticas gerenciais, não se mostraram relevantes antecedentes da confiança dos consumidores nessa dimensão do modelo proposto por Sirdeshmukh et al (2002), no contexto desta pesquisa. Nesse sentido, sugere-se que alguns elementos relacionais não foram considerados relevantes pelos consumidores de farmácias da amostra desta pesquisa.

Dessa forma, a benevolência operacional (a situação visível na qual a organização e/ou seus funcionários colocam os interesses do consumidor acima dos próprios interesses) e a orientação para solução de problema (a predisposição para antecipar ou resolver problemas durante e/ou após uma troca de serviços) apresentaramse irrelevantes neste trabalho.

\section{Tabela 2}

Caracterização da amostra

\begin{tabular}{lcclcc}
\hline Faixa etária & Respondentes & $\%$ & Gênero & Respondentes & $\%$ \\
\hline 16 a 25 & 105 & $39,03 \%$ & Masculino & 129 & $47,96 \%$ \\
\hline 26 a 35 & 94 & $34,94 \%$ & Feminino & 140 & $52,04 \%$ \\
\hline 36 a 45 & 32 & $11,90 \%$ & & 269 & $100,00 \%$ \\
\hline 46 a 55 & 23 & $8,55 \%$ & & & \\
\hline 56 ou mais & 15 & $5,58 \%$ & & & \\
\hline
\end{tabular}

\begin{tabular}{lcc}
\hline Renda & Respondentes & $\%$ \\
\hline Até $\mathrm{R} \$ 1.000,00$ & 145 & $53,90 \%$ \\
\hline Entre $\mathrm{R} \$ 1.000,01$ a $\mathrm{R} \$ 2.000,00$ & 74 & $27,51 \%$ \\
\hline Entre $\mathrm{R} \$ 2.000,01$ a $\mathrm{R} \$ 3.000,00$ & 30 & $11,15 \%$ \\
\hline Acima de $\mathrm{R} \$ 3.000$ & 20 & $7,44 \%$ \\
\hline
\end{tabular}

Nota. Fonte: Resultados da pesquisa. Elaborado pelos autores.

A hipótese 8 (H8) também foi confirmada e comprova que a confiança nos funcionários de contato é importante para a confiança do consumidor nas políticas e práticas gerenciais. Da mesma forma, no trabalho de Sousa et al (2006), a confiança nos funcionários de contato tem maior influência na confiança nas políticas e práticas gerenciais do que o contrário. Por sua vez, em Porto (2004), a maior influência foi encontrada em sentido oposto.

Já neste estudo, a hipótese 7 (H7) foi rejeitada e, assim, a confiança nas políticas e práticas gerenciais não leva à confiança nos funcionários de contato. Os resultados do trabalho de Sousa et al (2006), em ordem de influência, foram iguais ao de Sirdeshmukh et al (2002) nos dois contextos norte-americanos pesquisados, no que tange à relação mútua das duas facetas da confiança. Da mesma maneira, Brei (2001), em estudo realizado com clientes usuários de internet banking, confirmou a importância de confiar nos funcionários para, consequentemente, confiar na empresa e suas políticas, corroborando com o resultado deste estudo.

Da mesma forma, a hipótese 11 (H11) foi confirmada, demonstrando que a percepção de valor é relevante para as intenções de lealdade dos clientes de farmácias de Paranaíba. Nesse sentido, Sousa et al (2006) afirmam que a elevada percepção de valor é fundamental para as intenções de lealdade dos 
consumidores. Isto se confirma na afirmação de Porto (2004) de que o impacto do valor percebido sobre a lealdade tem se mostrado forte em diversos estudos realizados.

Tabela 3

Coeficientes Estimados (não-padronizados e padronizados e significâncias estatísticas)

\begin{tabular}{|c|c|c|c|c|c|c|c|c|}
\hline & & & & & $\begin{array}{l}\text { ession } \\
\text { ghts }\end{array}$ & & & $\begin{array}{c}\text { Standardized } \\
\text { Regression Weights }\end{array}$ \\
\hline Hipótese & Situação & & & & & $\begin{array}{l}t- \\
\text { value }\end{array}$ & $\mathbf{P}$ & $\beta$ \\
\hline $\mathrm{H} 2$ & Confirmada & $\mathrm{CP}$ & $<---$ & PCO & 0,414 & 3,826 & $* * *$ & 0,201 \\
\hline $\mathrm{H} 4$ & Rejeitada & $\mathrm{CP}$ & $<---$ & PBO & 0,115 & 1,142 & $0,254^{a}$ & 0,061 \\
\hline $\mathrm{H} 6$ & Rejeitada & $\mathrm{CP}$ & $<---$ & POS & 0,172 & 1,867 & $\begin{array}{r}0,062 \\
a\end{array}$ & 0,099 \\
\hline $\mathrm{H} 1$ & Confirmada & $\mathrm{CF}$ & $<--$ & $\mathrm{FCO}$ & 0,515 & 4,312 & $* * *$ & 0,266 \\
\hline $\mathrm{H} 3$ & Rejeitada & CF & $<---$ & FBO & 0,151 & 1,078 & $\begin{array}{r}0,281 \\
a\end{array}$ & 0,060 \\
\hline $\mathrm{H} 5$ & Rejeitada & $\mathrm{CF}$ & $<<--$ & FOS & 0,275 & 2,827 & $0,05^{a}$ & 0,156 \\
\hline $\mathrm{H} 12$ & Confirmada & VAL & $<--$ & $\mathrm{CF}$ & 0,260 & 4,186 & $* * *$ & 0,310 \\
\hline $\mathrm{H} 13$ & Confirmada & VAL & $<--$ & $C P$ & 0,375 & 5,215 & $* * *$ & 0,386 \\
\hline $\mathrm{H} 10$ & Rejeitada & LEAL & $<---$ & $\mathrm{CP}$ & 0,176 & 2,046 & $\begin{array}{r}0,041 \\
a\end{array}$ & 0,160 \\
\hline $\mathrm{H} 9$ & Rejeitada & LEAL & $<---$ & $\mathrm{CF}$ & $-0,028$ & $-0,381$ & $\begin{array}{r}0,703 \\
\mathrm{a}\end{array}$ & $-0,029$ \\
\hline $\mathrm{H} 11$ & Confirmada & LEAL & $<---$ & VAL & 0,632 & 9,071 & $* * *$ & 0,558 \\
\hline $\mathrm{H} 7$ & Rejeitada & $\mathrm{CF}$ & $<---$ & $\mathrm{CP}$ & 0,369 & 2,835 & $0,05^{a}$ & 0,318 \\
\hline $\mathrm{H} 8$ & Confirmada & $\mathrm{CP}$ & $<--$ & $\mathrm{CF}$ & 0,424 & 5,243 & $* * *$ & 0,492 \\
\hline
\end{tabular}

Nota. Fonte: Resultados da Pesquisa. Elaborado pelos autores.

*** Valores altamente significativos $p<0,001$; $a-p>0,05$ (valores sem significância estatística); Os valores de $t$-value são os mesmos para os coeficientes padronizados e não padronizados.

CP - Confiança nas Políticas e Práticas Gerenciais; CF - Confiança nos Funcionários de Contato; VAL - Valor; LEAL - Lealdade. PCO, PBO e POS - Competência Operacional, Benevolência Operacional e Orientação para Solução de Problemas de Políticas e Práticas Gerenciais. FCO, FBO e FOS - Competência Operacional, Benevolência Operacional e Orientação para Solução de Problemas de Funcionários de Contato

Outra hipótese confirmada foi a H12, corroborando a proposição do modelo de Sirdeshmukh et al (2002) de que a confiança nos funcionários de contato tem relação com a percepção de valor do consumidor. Da mesma maneira, a hipótese 13 (H13) foi confirmada, ratificando que a confiança nas políticas e práticas gerenciais contribui para percepção de valor.

Estes resultados demonstraram que o valor percebido tem papel relevante para as intenções de lealdade dos consumidores de farmácias em Paranaíba, bem como, é também um importante mediador entre as duas facetas da confiança e a lealdade, como proposto pelo modelo de Sirdeshmukh et al (2002). A confiança nas políticas e práticas gerenciais e a confiança nos funcionários de contato não exerceram influência direta sobre a lealdade, somente indiretamente, ao exercerem influência positiva e significativa sobre o valor.

Assim, sete das treze hipóteses deste estudo foram rejeitadas. Dessa maneira, o modelo proposto por Sirdeshmukh et al (2002) conseguiu, mesmo que parcialmente, descrever as relações entre confiança, valor e lealdade nas transações entre farmácias e consumidores na cidade de Paranaíba.

\section{Conclusões}

Os resultados da pesquisa apontaram um bom ajustamento do modelo proposto por Sirdeshmukh et al (2002) ao contexto do varejo farmacêutico da cidade de Paranaíba, MS. A amostra levantada foi caracterizada, em sua maioria, por jovens, do gênero feminino e com renda máxima de mil reais.

A competência operacional dos funcionários de contato apresentou-se como fator relevante para que os consumidores confiem nesses mesmos funcionários. Da mesma forma, confiar nos funcionários de contato mostrou-se relevante para que os consumidores confiem nas políticas e práticas gerenciais da farmácia.

Dessas interdependências pode-se concluir que os funcionários desempenham papel relevante no desenvolvimento da confiança dos consumidores nas farmácias com as quais se relacionam. Possivelmente, o contexto da pequena cidade do interior do estado amplifique o papel exercido pelos funcionários de contato. Isso reforça o que afirma Reichheld (2002), ou seja, de que a lealdade dos funcionários deve ser conquistada e não somente a dos clientes, pois cabe ao funcionário um importante papel no desenvolvimento de relacionamentos bem sucedidos. Mattila (2001) também afirma que as estratégias de marketing de relacionamento devem bem cuidar das interações entre consumidores e funcionários. 
A competência operacional foi único antecedente a exercer influência sobre a confiança em suas duas facetas: funcionários de contato e políticas e práticas gerenciais. Assim, a benevolência operacional e a orientação para solução de problemas não exerceram influência significativa sobre a confiança para a amostra levantada. Dessa forma, a afirmação de Porto (2004) e Sirdeshmukh et al (2002) de que a benevolência operacional é importante na explicação da confiança não se confirmou nos resultados deste trabalho. Da mesma maneira, a orientação para solução de problemas, ou seja, as motivações para antecipar e resolver problemas de forma satisfatória, não se apresentou como relevante antecedente da confiança.

Outra relevante constatação está no fato de que as duas facetas da confiança (políticas e práticas gerenciais e funcionários de contato) exerceram influência positiva e significativa sobre a percepção de valor dos consumidores de farmácias da cidade de Paranaíba, MS. Assim, como propõem Sirdeshmukh et al (2002), verifica-se que existe um relevante papel da confiança na formação do valor percebido pelo consumidor. Todavia, em nenhuma das duas facetas foi verificada qualquer relação entre a confiança e a lealdade do consumidor, sendo essa uma das relações fundamentais na proposta de Sirdeshmukh et al (2002).

Conforme afirmam autores como Sousa et al (2006) e Porto (2004), a percepção de valor tem se relacionado fortemente com as intenções de lealdade em distintos setores. Neste trabalho não foi diferente. Ao perceber que os benefícios são superiores a todo tipo de custo envolvido no relacionamento com uma organização, o consumidor tende a estabelecer com a mesma um relacionamento duradouro e a se comportar de maneira a refletir suas intenções de lealdade.

Contudo, sete das treze hipóteses propostas para a pesquisa foram rejeitadas e apontam para a necessidade de repensar o modelo para o varejo farmacêutico. Dessa forma, no contexto desta pesquisa, o papel dos funcionários de contato parece mais visível ao consumidor do que o papel das políticas e práticas gerenciais.

Resumidamente, neste estudo observou-se a seguinte relação: a competência operacional dos funcionários de contato leva à confiança nesses mesmos funcionários. Por sua vez, a confiança nos funcionários de contato leva a valor e à confiança nas políticas e práticas gerenciais. A competência operacional, expressa nas políticas e práticas gerenciais, leva à confiança do consumidor nas políticas e práticas gerenciais da organização e, por sua vez, tal confiança relaciona-se com o valor percebido. Na última relação significativa, tem-se que valor leva a lealdade.

Uma relevante contribuição acadêmica foi apresentada ao se estudar o varejo farmacêutico sob a perspectiva do marketing de relacionamento e, mais especificamente, sobre os construtos que antecedem a formação da lealdade do consumidor. O varejo farmacêutico, como verificado no levantamento bibliográfico, ainda carece de estudos nessa direção. Como consequência, verificou-se que o modelo das inter-relações entre confiança, valor e lealdade, que obteve bom ajustamento aos dados obtidos, explicou apenas parcialmente as relações teóricas propostas, para a amostra e o contexto em questão.

Como contribuição gerencial, salienta-se que as farmácias de Paranaíba, MS, devem ter especial cuidado em relação aos aspectos da competência operacional, como a rapidez e eficiência no atendimento e na entrega dos produtos, fundamental para consumidores de medicamentos; a qualificação dos funcionários para o atendimento e a orientação adequada às demandas desses consumidores; além da limpeza e organização do estabelecimento. Essas questões são relacionadas diretamente à competência operacional, antecedente que se mostrou relevante na formação da confiança do consumidor. Bem como, cabe aos gerentes identificar os potenciais clientes relacionais e adotar estratégias para conquistar sua lealdade, o que contribuirá, de tal maneira, para o aumento da competitividade da organização, obtendo benefícios como a recompra e a espontânea decisão de promover a empresa junto a amigos e familiares, por meio do boca a boca positivo.

Como limitação, propõe-se aumentar o tamanho da amostra em trabalhos futuros, levando-se em consideração as recomendações de Hair et al (1995) para utilizar-se de 5 a 15 respondentes por parâmetro a ser estimado no modelo. Neste trabalho foram estimados 48 parâmetros e foi obtida uma amostra de 269 respondentes, o que representa cerca de 5,6 respondentes/parâmetro estimado, atendendo ao sugerido por Hair et al (1995). Todavia, Hair et al (1995) salientam que o número de 15 respondentes por parâmetro estimado deve ser utilizado para que se corrijam possíveis distorções que podem ser ocasionadas por dados que não apresentam normalidade multivariada. Outra limitação está no próprio contexto da pesquisa: cidade pequena, do interior de um estado novo e pouco povoado, com um grau de concorrência comparativamente baixo em relação aos maiores centros urbanos. Sugere-se, também, que futuras aplicações do modelo de Sirdeshmukh et al (2002), no varejo farmacêutico, as amostras sejam obtidas por meio de amostragem probabilística, valendo-se de uma amostragem aleatória sistemática, na qual “a população-alvo definida seja ordenada de algum modo, em geral na forma de uma lista de clientes [ ...]" (Hair et al, 2010, p. 160).

Sugere-se que, na proposição de novos modelos para o varejo farmacêutico, a divisão da confiança em duas facetas seja repensado, uma vez que neste estudo não se confirmaram relações importantes entre as duas facetas da confiança com a lealdade do consumidor. Em virtude da força apresentada pelo valor sobre as intenções de Lealdade, sugere-se ainda que estudos exploratórios possam investigar questões como localização e preço, além da identificação e distinção dos consumidores de fato propensos à lealdade dos consumidores meramente transacionais nesse segmento. Reforça-se tal sugestão pelo fato da confiança não ter apresentado qualquer relação com as intenções de lealdade dos consumidores nesta pesquisa. 
Por fim, as relações entre confiança, valor e lealdade foram, mesmo que parcialmente, confirmadas no contexto do varejo farmacêutico da cidade de Paranaíba, MS, confirmando, dessa maneira, em mais um contexto, a qualidade do modelo proposto por Sirdeshmukh et al (2002).

\section{Referências}

Almeida, T. N. V., \& Ramos, A. S. M. (2012). Os impactos das reclamações on line na lealdade dos consumidores: um estudo experimental. Revista de Administração Contemporânea, 16(5), 664-683.

Arbuckle, J. L. (1999). Amos User's Guide - Version 4.0. Chicago: SPSS.

Bagozzi, R. P., \& Edwards, J. R. (1998). A general approach for representing constructs in organizational research. Organizational Research Methods, 1(1), 45-87.

Bateson, J., \& Hoffman, D. (2001). Marketing de serviços. Porto Alegre: Bookman.

Bei, L., \& Chiao, Y. (2001). An Integrated Model for the Effects of Perceived Product, Perceived Service Quality, and Perceived Price Fairness on Consumer Satisfaction and Loyalty. Journal of Consumer Satisfaction, Dissatisfaction and Complaining Behavior, 14, 125-140.

Berndt, A., \& Oliveira, L. H. (2005). A Construção do Saber Administrativo por Meio de Replicagens em Pesquisas por Levantamento (survey), Revista ANGRAD. 6(3), 9-26.

Berry, L. (2002). Relationship Marketing of Services - Perspectives from 1983 and 2000. Journal of Relationship Marketing, 1(1), 59-77.

Bitner, M. Booms, B., \& Mohr, L. (2001). Encontros críticos de serviços: o ponto de vista do funcionário. In: J. Bateson \& D, HOFFMAN, D. Marketing de services (pp. 112-125). Porto Alegre: Editora Bookman.

Brei, V. (2001). Antecedentes e Consequências da Confiança do Consumidor Final em Trocas Relacionais com Empresas de Serviço: um estudo com o usuário de Internet Banking no Brasil. Dissertação de mestrado, Universidade Federal do Rio Grande do Sul, Porto Alegre, RS, Brasil.

Churchill, G., \& Peter, J. (2000). Marketing. Criando valor para os clientes. São Paulo: Saraiva.

Doney, P., \& Cannon, J. (1997). An Examination of the Nature of Trust in Buyer-Seller Relationships. Journal of Marketing, 61(1), 35-51.

Engel, J., Blackwell, R., Miniard, P. (2000). Comportamento do Consumidor. Rio de Janeiro: LTC Editora.

Garbarino, E., \& Johnson, M. (1999). The Different Roles of Satisfaction, Trust, and Commitment in Customer Relationships. Journal of Marketing, 63(2), 70-87.

Grönroos, C. (1994). From Marketing Mix to Relationship Marketing: Towards a Paradigm Shift in Marketing. Management Decision, 32(1), 04-20.

Hair, J. Anderson, R. Tatham R., \& Black, W. (1995). Multivariate Data Analysis with Readings. 4. ed. New Jersey: Prentice Hall.

Hair, Joseph F. Jr., Wolfinbarger, Mary, Ortinau, David J., \& Bush, Robert P. (2010). Fundamentos de Pesquisa de Marketing. Porto Alegre: Bookman.

Hennig-Thurau, T. Gwinner, K., \& Gremler, D. (2002). Understanding Relationship Marketing Outcomes. Journal of Service Research, 4(3), 230-247.

Jap, S. (2001). The Strategic Role of the Sales force in Developing Customer Satisfaction Across the Relationship Lifecycle. Journal of Personal Selling and Sales Management, 21(2), 95-108.

Kleinowski, H. L. (2009). O estudo da confiança, valor percebido e lealdade do segmento farmacêutico brasileiro. Gestão Contemporânea, 6(6), 121-145.

Lovelock, C., \& Wright, L. (2002). Serviços: Marketing e Gestão. São Paulo: Saraiva.

Malhotra, N. (2001). Pesquisa de Marketing: Uma Orientação Aplicada. Porto Alegre: Bookman.

Marôco, J. (2010). Análise de equações estruturais: fundamentos teóricos, software \& aplicações. Pêro Pinheiro: ReportNumber.

Mattila, A. (2001). The Impact of Relationship Type on Customer Loyalty in a Context of Service Failures. Journal of Service Research, 4, 91-101.

Maxham III, J, \& Netemeyer, R. (2002). A Longitudinal Study of Complaining Customers Evaluations of Multiple Service Failures and Recovery Efforts. Journal of Marketing, 66 (1), 57-71.

Murray, K. (1991). A Test of Services Marketing Theory: Consumer Information Acquisition Activities. Journal of Marketing, 55(1), 10-25.

Oliver, R. (1997). Satisfaction: A Behavioral Perspective on the Consumer. New York: The McGraw-Hill Companies. 
Oliver, R. (1999). Whence Consumer Loyalty? Journal of Marketing. 63 (Special Issue), 33-44.

Porto, C. A. (2004) As Relações entre Confiabilidade, Confiança, Valor e Lealdade no Contexto de Educação a Distância. Dissertação de mestrado, Pontifícia Universidade Católica do Rio Grande do Sul, Porto Alegre, RS, Brasil.

Reichheld, F., \& Schefter, P. (2000). E-Loyalty: Your Secret Weapon on the Web. Harvard Business Review, 784), 105-113.

Reichheld, F. (2002). Princípios da Lealdade: como os líderes atuais constroem relacionamentos duradouros e lucrativos. Rio de Janeiro: Campus.

Reichheld, F. (1996). A estratégia da lealdade: a força invisível que mantém clientes e funcionários e sustenta crescimento, lucros e valor. Rio de Janeiro: Campus.

Rowley, J., \& Dawes, J. (2000). Disloyalty: a closer look at non-loyals. Journal of Consumer Marketing, 17(6), 538549.

Schwab, E. A. (2009). As relações entre confiança, valor e lealdade: um estudo qualitativo. Dissertação de mestrado, Pontifícia Universidade Católica do Rio Grande do Sul, Porto Alegre, RS, Brasil.

Singh, J., \& Sirdeshmukh, D. (2000). Agency and Trust Mechanisms in Consumer Satisfaction and Loyalty Judgments. Journal of the Academy of Marketing Science, 28(1), 150-167.

Sirdeshmukh, D. Singh, J., \& Sabol, B. (2002). Consumer Trust, Value, and Loyalty in Relational Exchanges. Journal of Marketing, 66(1), 15-37.

Sousa, D. Rezende, D. C., \& Oliveira, L. H. (2006). As relações entre confiança, valor e lealdade: um estudo de caso em uma universidade federal. Anais do Encontro Nacional dos Programas de Pós-Graduação em Administração, Salvador, BA, Brasil, 30.

Zeithaml, V. (1988). Consumer Perceptions of Price, Quality and Value: A Means-End Model and Synthesis of Evidence. Journal of Marketing, 52(3), 2-22.

Zeithaml, V. Parasuraman, A., \& Berry, L. L. (1990). Delivering Quality Service: Balancing Customer Perceptions and Expectations. New York: The Free Press. 